\title{
Abstracts from Nippon Eiseigaku Zasshi (Japanese Journal of Hygiene) vol. 70, no. 2
}

(C) The Japanese Society for Hygiene 2015

\section{Analysis of environmental-stress-related impairments of inner ear}

Nippon Eiseigaku Zasshi, 70, 100-104 (2015)

Nobutaka Ohgami $^{1,2}$, Machiko Iida ${ }^{1,2}$, Yasuhiro Omata ${ }^{1}$, Chihiro Nakano ${ }^{1}$, Wu Wenting ${ }^{1}$, Xiang $\mathrm{Li}^{1}$, Masashi Kato ${ }^{1}$

${ }^{1}$ Department of Occupational and Environmental Health, Nagoya University Graduate School of Medicine

${ }^{2}$ Nutritional Health Science Research Center, Chubu University

Noise stress generated in industry is one of the environmental factors that physically affects the functions of the inner ear. Exposure to noise can cause hearing loss, resulting in serious problems in occupational and daily life. At present, however, there are very limited ways to prevent hearing impairments. The inner ear consists of the organ of Corti, vestibule and semicircular canal. Functional or morphological damage of these tissues in the inner ear caused by genetic factors, aging or environmental factors can result in hearing or balance impairments. In this review, we first introduce a deafness-related molecule found by our clinical research. Our experimental research using genetically engineered mice further demonstrated that impaired activity of the target molecule caused congenital and age-related hearing loss with neurodegeneration of spiral ganglion neurons in the inner ears. We also describe impaired balance in mice caused by exposure to low-frequency noise under experimental conditions with indoor environmental monitoring. We believe that our approaches to pursue both experimental research and fieldwork research complementarily are crucial for the development of a method for prevention of impairments of the inner ear.

\section{Analysis of heavy-metal-mediated disease and development of a novel remediation system based on fieldwork and experimental research}

Nippon Eiseigaku Zasshi, 70, 105-109 (2015)

Ichiro Yajima, Cunchao Zou, Xiang Li, Chizuru Nakano, Yasuhiro Omata, Mayuko Y. Kumasaka

Department of Occupational and Environmental Health, Nagoya University Graduate School of Medicine
Heavy-metal pollution occurs in various environments, including water, air and soil, and has serious effects on human health. Since heavy-metal pollution in drinking water causes various diseases including skin cancer, it has become a global problem worldwide. However, there is limited information on the mechanism of development of heavy-metal-mediated disease. We performed both fieldwork and experimental studies to elucidate the levels of heavymetal pollution and mechanisms of development of heavy-metal-related disease and to develop a novel remediation system. Our fieldwork in Bangladesh, Vietnam and Malaysia demonstrated that drinking well water in these countries was polluted with high concentrations of several heavy metals including arsenic, barium, iron and manganese. Our experimental studies based on the data from our fieldwork demonstrated that these heavy metals caused skin cancer and hearing loss. Further experimental studies resulted in the development of a novel remediation system with which toxic heavy metals were absorbed from polluted drinking water. Implementation of both fieldwork and experimental studies is important for prediction, prevention and therapy of heavy-metal-mediated diseases.

\section{Experimental approach to analysis of the relationship between food environments and lifestyle-related diseases, including cardiac hypertrophy, fatty liver, and fatigue symptoms}

Nippon Eiseigaku Zasshi, 70, 110-114 (2015)

Masahisa Horiuchi ${ }^{1}$, Miwa Nakakuma ${ }^{1,2}$, Emi Arimura ${ }^{1,3}$, Miharu Ushikai ${ }^{1}$, Goichiro Yoshida ${ }^{1,4}$

${ }^{1}$ Department of Hygiene and Health Promotion Medicine, Kagoshima University Graduate School of Medical and Dental Sciences

${ }^{2}$ Takata Hospital (Kagoshima City)

${ }^{3}$ Major in Food and Nutrition, Department of Life and Environmental Science, Kagoshima Prefectural College

${ }^{4}$ Department of Sports and Life Science, National Institute of Fitness and Sports in Kanoya

The food habit is involved in the onset and development of lifestylerelated diseases. In this review I would like to describe a historical case of vitamin $B_{1}$ deficiency, as well as our case study of fatty acid metabolism abnormality due to carnitine deficiency. In history, the army and 
navy personnel in Japan at the end of the 19th century received food rations based on a high-carbohydrate diet including white rice, resulting in the onset of beriberi. An epidemiological study by Kenkan Takaki revealed the relationship between the onset of beriberi and rice intake. Then, Takaki was successful in preventing the onset of beriberi by changing the diet. However, the primary cause had yet to be elucidated. Finally, Christian Eijkman established an animal model of beriberi (chickens) showing peripheral neuropathy, and he identified the existence of an anti-beriberi substance, vitamin $B_{1}$. This is an example of the successful control of a disease by integrating the results of epidemiological and experimental studies. In our study using a murine model of fatty acid metabolism abnormality caused by carnitine deficiency, cardiac abnormality and fatty liver developed depending on the amount of dietary fat. In addition, the mice showed disturbance of orexin neuron activity related to the sleep-arousal system, which is involved in fatigue symptoms under fasting condition, one of the states showing enhanced fatty acid metabolism. These findings suggest that fatty acid toxicity is enhanced when the mice are more dependent on fatty acid metabolism. Almost simultaneously, a human epidemiological study showed that narcolepsy, which is caused by orexin system abnormality, is associated with the polymorphism of the gene coding for carnitine palmitoyltransferase $1 \mathrm{~B}$, which is involved in carnitine metabolism. To understand the pathological mechanism of fatty acid toxicity, not only an experimental approach using animal models, but also an epidemiological approach is necessary. The results will be applied to preventing and treating lifestyle-related diseases associated with fatty acid metabolism abnormality.

\section{Immune system reaction against environmental pollutants}

Nippon Eiseigaku Zasshi, 70, 115-119 (2015)

Tsuyoshi Tanabe, Natsu Yamaguchi, Masayuki Okuda, Yasutaka Ishimaru, Hidekazu Takahashi

Department of Public Health, Yamaguchi University Graduate School of Medicine

Environmental pollutants (such as diesel exhaust particles and silica) cause disorders ranging from bronchial asthma to malignant tumors. In recent years, it has been reported that some of the signaling pathways in which environmental contaminants act in vivo are associated with innate immunity. Innate immunity recognizes ligands and induces inflammation. Those ligands are pathogen-associated molecular patterns (PAMPs: e.g., lipopolysaccharide) and danger-associated molecular patterns (DAMPs: e.g., cholesterol crystallization or uric acid crystal). Activation of innate immunity stimulates the acquired immunity system. Therefore, innate immunity regulates the strength of the general immune system. Furthermore, crystal silica, which is an environmental pollutant, activates innate immunity as a ligand. Innate immunity involves the membrane-bound Toll-like receptors (TLR) and cytoplasm-localized nucleotide-binding oligomerization domain (NOD)-like receptors (NLR). We reported the innate immunity-system-related diseases such as Crohn's disease, Blau syndrome, myelogenous leukemia, and sarcoidosis. An inflammasome complex containing NLR has attracted attention owing to its correlation with the onset of several diseases. It is reported that the inflammasome activation is related to the development of lifestyle-related diseases such as myocardial infarction and fatty liver. It is also reported that the mechanism by which crystal silica and asbestos cause inflammation involves the inflammasome activation. Analyzing the genes of innate immunity contributes to the clarification of the mechanism of disease onset caused by environmental pollutants.
Development of higher brain function tests in rodents and its application to neurotoxicity assessment of environmental chemicals

\author{
Nippon Eiseigaku Zasshi, 70, 120-126 (2015) \\ Masaki Kakeyama ${ }^{1,2}$ \\ ${ }^{1}$ Laboratory of Environmental Health Sciences, CDBIM, Graduate \\ School of Medicine, The University of Tokyo \\ ${ }^{2}$ Laboratory for Systems Neuroscience \& Preventive Medicine, \\ Waseda University Faculty of Human Sciences
}

The brain during developmental period is thought to be highly sensitive to environmental insults including exposure to chemicals. However, it has been extremely difficult to detect and assess the features and degree of adversity particularly at low exposure levels. I describe here the effects of maternal exposure to dioxin on higher brain functions later in life, which we detected using our originally developed behavioral tests for quantifying higher brain functions in rodents. We first found changes in the mRNA expression levels of glutamate NMDA receptor subunits that have critical roles in learning and memory function in the neocortex and hippocampus. To assess the neocortical and hippocampal functions in rats, we established novel behavioral tests for assessing paired-associate learning, which is the hippocampal and medial prefrontal NMDA-dependent function. Maternal exposure to dioxin, at a low level of which does not affect simple memory formation, resulted in the disturbance of the pairedassociate learning. On the basis of the above learning paradigm, we next developed a behavioral flexibility task and a social competitive task for mice using the automated behavioral assessment system 'IntelliCage': this system can accommodate 16 mice at the same time to monitor and record their behavior. Using this system, we found that male mice born to dams exposed to very low doses of dioxin showed inflexibility in a serial reversal learning task and socially low-dominance behavior under a competitive situation. Immunohistochemical analysis of putative neuronal activity markers revealed hypoactivity in the medial prefrontal cortex (mPFC) of dioxin-exposed mice. We speculate that $\mathrm{mPFC}$ hypoactivity reflects the dioxin-induced higher brain dysfunction and may be associated with some psychiatric illnesses and related problems. These behavioral tests were found to be useful for studying the higher brain functions of rats and mice.

\section{Oxidative stress derived from airborne fine and ultrafine particles and the effects on brain-nervous system: part 1}

Nippon Eiseigaku Zasshi, 70, 127-133 (2015)

Masaru Sagai ${ }^{1}$, Tin Tin Win-Shwe ${ }^{2}$

${ }^{I}$ Tsukuba Institute for Healthy Life (Aomori University of Health and Welfare)

${ }^{2}$ National Institute for Environmental Studies

Traffic-related air pollution is a major contributor to urban air pollution. Diesel exhaust (DE) is the most important component of nearroad and urban air pollution and is commonly used as a surrogate model of air pollution in health effects studies. In particular, diesel exhaust particles (DEP) and the nanoparticles in DEP are considered hazardous components on health effects. It is widely known that exposure to DEP is associated with mortality due to respiratory and cardiovascular diseases. Recently, there has been accumulating evidence that DEP and the nanoparticles in DEP may be causes of neurodegenerative disorders. Here, we introduce the evidence suggesting their association with such disorders. First, we describe the 
chemical components and the translocation of DEP and nanoparticles to the brain, and then introduce the evidence and a mechanism by which reactive oxygen species (ROS) and any inflammatory mediators can be produced by DEP phagocytosis of macrophages, microglia and astrocyte cells in the brain. There are many lines of evidence showing that the neurodegenerative disorders are profoundly associated with enhanced oxidative and inflammatory events. Second, we describe a mechanism by which neurodegenerative diseases, such as stroke, Alzheimer's disease and Parkinson's disease, are induced via oxidative stress and inflammatory events.

\section{Associations between $A L D H 2$ and $A D H 1 B$ genotypes and ethanol-induced cutaneous erythema in young Japanese women}

Nippon Eiseigaku Zasshi, 70, 134-138 (2015)

Mariko Hayashida, Yuka Kamada, Tomoko Ota, Sayuri Kojima, Kyoko Iwao-Koizumi, Shigenori Murata, Kenji Kinoshita

School of Pharmaceutical Sciences, Mukogawa Women's University

Objectives: The purpose of this study was to identify associations between $A L D H 2$ and $A D H 1 B$ genotypes and ethanol-induced cutaneous erythema and assess the accuracy of an ethanol patch test in young Japanese women.

Methods: The subjects were 942 female Japanese university students. They were given an ethanol patch test and examined for ethanol-induced cutaneous erythema both immediately after removing the patch and 10 min after removing the patch. A saliva sample was used to determine the $A L D H 2$ and $A D H 1 B$ genotype of each subject by realtime PCR.

Results: The sensitivity and specificity of erythema immediately after removing the patch as the marker for the presence of inactive ALDH2 were 69.6 and $87.7 \%$, respectively, and the sensitivity and specificity of erythema $10 \mathrm{~min}$ after removing the patch were 85.2 and $85.1 \%$, respectively. The sensitivity of erythema after 10 min was markedly lower in the $A D H 1 B * 1 / * 1$ carriers than in the $A D H 1 B * 2$ carriers ( 8.3 vs. $89.7 \%$, $p<0.0001$ ), and the specificity was significantly higher in the $A D H 1 B * 11$ $* 1$ carriers than in the $A D H 1 B * 2$ carriers (96.9 vs. $84.3 \%, p<0.05$ ).

Conclusions: Overall, both sensitivity and specificity were satisfactorily high, but having the $A D H 1 B * 1 / * 1$ genotype prevented a positive reaction for inactive ALDH2 and caused false-negative results. The data also suggested that having the $A D H 1 B * 2 / * 2$ genotype caused a positive reaction in subjects with the $A L D H 2 * 1 / * 1$ genotype. Despite these exceptions, the ethanol patch test has enough accuracy and can be used easily to subjects who don't drink alcohol. This is a valuable tool for improving the health literacy of younger generation subjects.

\section{Menstrual cycle length and source of its variation in female university students majoring in nursing sciences}

Nippon Eiseigaku Zasshi, 70, 139-148 (2015)

Yukiko Nishihama $^{1}$, Jun Yoshinaga ${ }^{1}$, Ayaka Iida ${ }^{2}$, Shoko Konishi $^{3,4}$, Hideki Imai ${ }^{5}$

${ }^{1}$ Department of Environmental Studies, University of Tokyo

${ }^{2}$ Department of Environmental, Occupational Health and Toxicology, School of Allied Health Sciences, Kitasato University

${ }^{3}$ Department of Human Ecology, Graduate School of Medicine, University of Tokyo

${ }^{4}$ Department of Anthropology, University of Washington

${ }^{5}$ Department of Nursing, Tokyo Health Care University
Objectives: To acquire fundamental knowledge on menstrual cycle and its intraindividual variation in healthy Japanese female students and their association with biological attributes, lifestyle and food habit.

Methods: We asked female students of a nursing school to keep a diary of their menstruation over 5 consecutive months and to fill a questionnaire on their biological attributes, lifestyle and consumption frequency of some food items during the period from November 2012 to January 2014.

Results: The mean \pm standard deviation (SD) of cycle lengths of 180 women $(20.4 \pm 0.9$ years $)$ was $34.2 \pm 10.5$ days. Of these 180 women, 141 had a cycle length within the normal range (25-38 days) while 10 had a shorter cycle and 20 had a longer cycle. The intraindividual variations of the cycle, defined as "range" (difference between the longest and shortest cycles of an individual) and "SD" (SD of mean of multiple lengths within an individual), were $11.4 \pm 10.3$ and $7.9 \pm 11.8$ days, respectively. Of 177 women, 73 and 109 had normal range and SD ( $\leq 6$ days), respectively. The mean age of women with long cycles was older than that of women with short cycle (Kruscal-Wallis test, $p<0.05)$, and women with greater intraindividual variations were older than those with small variations (U-test, $p<0.05$ ). Subjects with menstrual pain had greater "SD" than did those without pain (Chi square test, $p<0.05$ ). Neither lifestyle nor food habit was associated with the length and intraindividual variations of the menstrual cycle.

Conclusions: The present results provided fundamental knowledge on the length and intraindividual variations of the menstrual cycle and the sources of these variations in female Japanese university students.

\section{Measures against radiation exposure due to large-scale nuclear accident in distant place: radioactive materials in Nagasaki from Fukushima Daiichi nuclear power plant}

Nippon Eiseigaku Zasshi, 70, 149-160 (2015)

Jun Yuan ${ }^{1}$, Koichiro Sera ${ }^{2}$, Toshihiro Takatsuji ${ }^{1}$

${ }^{I}$ Graduate School of Fisheries Science and Environmental Studies, Nagasaki University

${ }^{2}$ Cyclotron Research Center, Iwate Medical University

Objective: To investigate human health effects of radiation exposure due to possible future nuclear accidents in distant places and other various findings of analysis of the radioactive materials contaminating the atmosphere of Nagasaki due to the Fukushima Daiichi Nuclear Power Plant accident.

Methods: The concentrations of radioactive materials in aerosols in the atmosphere of Nagasaki were measured using a germanium semiconductor detector from March 2011 to March 2013. Internal exposure dose was calculated in accordance with ICRP Publ. 72. Air trajectories were analyzed using NOAA and METEX web-based systems.

Results: ${ }^{134} \mathrm{Cs}$ and ${ }^{137} \mathrm{Cs}$ were repeatedly detected. The air trajectory analysis showed that ${ }^{134} \mathrm{Cs}$ and ${ }^{137} \mathrm{Cs}$ flew directly from the Fukushima Daiichi Nuclear Power Plant from March to April 2011. However, the direct air trajectories were rarely detected after this period even when ${ }^{134} \mathrm{Cs}$ and ${ }^{137} \mathrm{Cs}$ were detected after this period. The activity ratios $\left({ }^{134} \mathrm{Cs} /{ }^{137} \mathrm{Cs}\right)$ of almost all the samples converted to those in March 2011 were about unity. This strongly suggests that the ${ }^{134} \mathrm{Cs}$ and ${ }^{137} \mathrm{Cs}$ detected mainly originated from the Fukushima Daiichi Nuclear Power Plant accident in March 2011. Although the ${ }^{134} \mathrm{Cs}$ and ${ }^{137} \mathrm{Cs}$ concentrations per air volume were very low and the 
human health effects of internal exposure via inhalation is expected to be negligible, the specific activities (concentrations per aerosol mass) were relatively high.

Conclusion: It was found that possible future nuclear accidents may cause severe radioactive contaminations, which may require radiation exposure control of farm goods to more than $1000 \mathrm{~km}$ from places of nuclear accidents.

\section{Psychological effects of forest therapy program on workers}

Nippon Eiseigaku Zasshi, 70, 161-166 (2015)

Harumi Ikei ${ }^{1,4}$, Haruka Koizumi ${ }^{1,5}$, Chorong Song ${ }^{1}$, Mitsunori Kouzuki $^{2}$, Seiichiro Teratani ${ }^{2}$, Takahiro Sakuma ${ }^{3}$, Yoshifumi Miyazaki ${ }^{1}$

${ }^{1}$ Center for Environment, Health and Field Sciences, Chiba University

${ }^{2}$ Chizu Town Office

${ }^{3}$ LASSIC Co., Ltd.

${ }^{4}$ Current address: Forestry and Forest Products Research Institute

${ }^{5}$ Current address: Yokohama City Office

Objectives: To examine the psychological effects of forest therapy program on workers.

Methods: The subjective symptoms index, a shortened version of the profile of mood states (POMS), and a semantic differential (SD) method were used to measure the psychological effects.

Results: The evaluations were performed 3 days before, during, and 1, 3 , and 5 days after the forest therapy. The following results were obtained: (1) the subjective symptoms improved before breakfast and continued for 5 days; (2) the mood evaluated using POMS improved before breakfast and continued for 3 days; and (3) "comfortable," "relaxed," and "natural" feelings evaluated using the SD method were enhanced before breakfast, lunch, and dinner during forest therapy.

Conclusions: These results provided scientific evidence of the psychological effects of forest therapy program on workers.

\section{Oral health status of pregnant women in Kumamoto Prefecture: Kumamoto RAINBOW Project}

Nippon Eiseigaku Zasshi, 70, 167-172 (2015)

Sakura Chiga ${ }^{1}$, Takashi Ohba ${ }^{1}$, Junya Miyoshi ${ }^{1,2}$, Daisuke Tanoue ${ }^{4}$, Hiromi Kawase ${ }^{4}$, Takahiko Katoh ${ }^{3}$, Hidetaka Katabuchi ${ }^{1}$

${ }^{1}$ Department of Obstetrics and Gynecology, Faculty of Life Sciences, Kumamoto University
${ }^{2}$ Department of Obstetrics and Gynecology, Japanese Red Cross Kumamoto Hospital

${ }^{3}$ Department of Public health, Faculty of Life Sciences, Kumamoto University

${ }^{4}$ Kumamoto Dental Association

Objectives: As part of Kumamoto RAINBOW Project, which is a multifaceted implementation for the prevention of premature labor, we investigated pregnant women's oral health status and assessed the effects of dental care and oral hygiene instruction.

Methods: We examined the oral health status of pregnant women both in the first and the second half of pregnancy in Kumamoto Prefecture from 2012 to 2014. The Community Periodontal Index (CPI) was used to assess the periodontal condition, and women having periodontal pockets with a depth $\geq 4 \mathrm{~mm}$ were defined as suffering from periodontitis. This project covered the cost of dental checkups.

Results: Of the 20,702 pregnant women enrolled in this project, 9527 (46.0\%) received dental checkups during the first half of pregnancy. The response rate of dental examinations in Kumamoto City $(63.3 \%)$, the capital city of Kumamoto Prefecture, was significantly higher than that of the other local areas $(32.0 \%)$. In Kumamoto City, 4890 women $(83.4 \%)$ had dental examinations at the city office when they received a maternal handbook. Three thousand forty-five women $(32.0 \%)$ had periodontitis. Among 1605 women who received oral examinations twice at dental clinics, 698 received nonsurgical interventions. Dental interventions significantly decreased the prevalence of periodontitis in pregnant women (55.1-45.1\%). Dental examinations without interventions also significantly decreased the prevalence of periodontitis (44.6-39.9\%).

Conclusions: Pregnant women living in Kumamoto City had higher rate of visits to dental clinics for checkups than those in other areas. Periodontitis was found in one-third of pregnant women. Not only dental interventions, but also dental examinations improve pregnant women's oral health status. 